EGU2020-3358

https://doi.org/10.5194/egusphere-egu2020-3358

EGU General Assembly 2020

(c) Author(s) 2020. This work is distributed under

the Creative Commons Attribution 4.0 License.

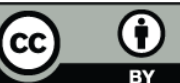

\title{
Towards a dynamic landslide susceptibility assessment: evaluation of a novel climate-related variable
}

\author{
Irene Corno, Corrado Camera, Greta Bajni, Stefania Stevenazzi, and Tiziana Apuani \\ Università degli Studi di Milano, Dipartimento di Scienze della Terra 'A. Desio', Italy (irene.corno@studenti.unimi.it)
}

The Mont Cervin and Mont Emilius Mountain Communities (Aosta Valley, North-West Italy) are particularly predisposed to shallow landslide phenomena due to their morphological and geological characteristics. In addition, short intense rainfalls, which are considered one of the main landslides triggering factors, are expected to increase over the Alpine region due to climate changes. This study was carried out to provide a potentially dynamic landslide susceptibility map, adaptable to these changes, for the two Communities (total area $670 \mathrm{~km}^{2}$ ). To achieve this goal, the susceptibility analysis was set up on a statistical basis, using the Logistic Regression method. The objectives of this study were:

- to verify the completeness of the database of dated shallow landslides, and define an optimal training set with the addition of non-landslide points;

- to find a potentially dynamic variable, statistically and physically significant, which summarizes the landslide-climate relationships;

- to derive a parsimonious model for the definition of landslide susceptibility that includes this variable.

For the period 1990-2018, 293 dated records of shallow landslides were extracted from the Landslide Regional Database. For non-landslide points, two sampling algorithms (Random and Stratified Sampling) and different sample sizes (from a minimum of one to a maximum of three times the number of landslide points) were evaluated. For the same period, the precipitation and temperature data were obtained from the time series available in Regional archives. The relationships between the triggering of landslides and the characteristics of the preceding precipitation (e.g., amount and intensity for durations ranging from 0.5 hours to 30 days) were studied using graphs and correlation indices, to determine the climatic variable to be used in the statistical analysis. Other geological-environmental data (e.g. elevation, land use, lithology) were downloaded from the Regional geoportal and then processed in a GIS environment to obtain traditional predictive variables. Logistic Regression analysis was implemented in SPSS. The models were evaluated through the confusion matrix, optimized keeping only the statistically significant variables, and validated through a 70\% (training) - 30\% (test) subdivision of the input data and the calculation of the Area Under the Curve (AUC values). The climatic variable was expressed in terms of the average annual number of exceedances of a rainfall intensity-duration landslide-triggering threshold, validated for the study area. The optimal sample of non-landslide points was obtained 
through Random Sampling and is equal to 1.15 times the number of landslide points. Statistically significant predictors were altitude, land use, slope and exceedances of the threshold. Applying the optimized model (discriminating probability 0.5 ), the true positives reached the $89.6 \%$ and $88.9 \%$ on training and test points, respectively. The resulting AUC values for the training and test curves are $83.1 \%$ and $82.1 \%$, respectively. Both indicators show that the model is robust and has good predictive power. The susceptibility map obtained from the developed model was reclassified through the geometrical interval method and $93 \%$ of the landslides fell into the high and very high susceptibility classes. 